\title{
Full sequence analysis and characterization of the South Korean Norovirus GII-4 variant CUK-3
}

\author{
Jeong-Woong Park', Sung-Geun Lee ${ }^{2}$, Young-Min Lee ${ }^{3}$, Weon-Hwa Jheong ${ }^{4}$, Sangryeol Ryu ${ }^{1 *}$ and \\ Soon-Young Paik ${ }^{2^{*}}$
}

\begin{abstract}
Background: Many of researchers have focused on the emerging pathogen, Norovirus, since its first identification as the causing agent of nonbacterial acute gastroenteritis in humans. One of the virulence factors of norovirus, the great genetic diversity attributed to point mutations and recombinations, has brought forth the result of significant changes in the circulating norovirus genotype patterns.

Findings: In recognition of the necessity for tracking and monitoring of genetic diversity, a norovirus variant among the most prevalent genotype Gll-4, Norovirus Hu/GIl-4/CUK-3/2008/KR (CUK-3), was isolated from stool samples and analyzed on the level of whole genome sequence. Whole genome sequence analysis revealed three ORF composites of the whole genome, ORF1 (5100 bp), ORF2 (1623 bp), and ORF3 (807 bp). Each genetic relationship of CUK-3 variant analysis located the ORF1 (5,100 bp) in Cluster I, ORF2 (1623 bp) in Cluster I (2006b), ORF3 (807 bp) in Cluster I, and the whole genome sequence (about $5.1 \mathrm{~kb}$ ) in Cluster I in the phylogenetic tree. And the phylogenetic analyses showed the same location of CUK-3 strain with the GII-4/2006b cluster in the phylogenetic tree.

Conclusions: In This study, a first concerning the full-length sequence of a NoV variant in South Korea is meaningful in that it can be used not only as a full-length NoV variant sequence standard for future comparison studies, but also as useful material for the public health field by enabling the diagnosis, vaccine development, and prediction of new emerging variants.
\end{abstract}

Keywords: Norovirus Sequence, Phylogenetic tree

\section{Findings}

Noroviruses (NoVs) are the most important viruses that cause nonbacterial acute gastroenteritis in humans. In addition to increased susceptibility, the elderly are at increased risk for more severe disease and death, as are the very young and the immunocompromised $[1,2]$. They are small, and non-enveloped viruses which and belong to the family Caliciviridae, genus Norovirus. Noroviruses have a single positive-strand NoV RNA genome of about $7.6 \mathrm{~kb}$ in size. Three open reading

\footnotetext{
* Correspondence: sangryu@snu.ac.kr; paik@catholic.ac.kr

'Department of Food and Animal Biotechnology, Department of Agricultural Biotechnology, Center for Agricultural Biomaterials, and Research Institute for Agriculture and Life Sciences, Seoul National University, Seoul 151-921,

Republic of Korea

2Department of Microbiology, College of Medicine, The Catholic University of Korea, Seoul 137-701, Republic of Korea

Full list of author information is available at the end of the article
}

frames (ORFs) have been identified in the NoV genomes. ORF1 encodes a polyprotein that is cleaved into six non-structural (NS) proteins, which carry amino acid sequence motifs conserved in NTPase, protease and RNA-dependent RNA polymerase (RdRp) $[3,4]$. ORF2 encodes a major structural protein, Viral Protein (VP1), which consist of two domains-the shell domain (S) and the protruding arm $(\mathrm{P})$ that is again divided into two subdomains, P1 and P2. The S domain is highly conserved while the $\mathrm{P}$ domain is variable. $\mathrm{P} 2$ of the $\mathrm{P}$ domain is hypervariable and carries immune and cellular recognition sites [5-7]. ORF3 encodes minor capsid protein, VP2, which is rich in basic amino acids and is proposed to have a role in viral stability $[8,9]$

Recently, NoVs were recognized as novel emergent pathogens. The main route of transmission is suspected to be person-to-person, but food and water-borne
C Biomed Central

(c) 2011 Park et al; licensee BioMed Central Ltd. This is an Open Access article distributed under the terms of the Creative Commons Attribution License (http://creativecommons.org/licenses/by/2.0), which permits unrestricted use, distribution, and reproduction in any medium, provided the original work is properly cited. 
transmission is also important $[1,10,11]$. According to nucleotide sequence analysis of the capsid regions, noroviruses are classified into five genogroups, GI to GV, each of which can be further divided into several clusters or genotypes [12]. Among the five genogroups, three genogroups (GI, GII and GIV) are known to cause clinical illness in humans, and genotype GII-4 has been the predominant circulating strain to the present [13]. The error-prone RNA replication and recombination between viruses is what drives noroviruses to its the great diversity. Furthermore, the accumulated mutations of the hypervariable P2 domain of the VP1 protein produced different GII-4 NoVs [14]. The most representative of the resulting variant GII-4 strain, GII-4/2006b, with 3 nucleotide insertions in the P2 domain at position 6265, emerged in the summer of 2002 which lead to a major gastroenteritis outbreak as well as an epidemic gastroenteritis worldwide in the winter of 2002/ 2003 [15,16]. In South Korea, gastroenteritis outbreaks by GII-4/2006b variants have been reported from September of 2007 to July of 2008 had been reported [17].

In this paper, the whole genome sequence of another isolated variant of the emerging strain type, the GII-4 variant, was analyzed and compared with other variants to reveal the genetic relationship and to predict the tendency of GII-4 variants in South Korea.

NoV positive-stool sample was isolated from patients with acute gastroenteritis in Daejeon, South Korea in November 2008. The sample was obtained from the Waterborne Virus Bank (Seoul, Korea). The stool sample was stored at $-70^{\circ}$. The viral genomic RNA was extracted from $140 \mu \mathrm{l}$ of $10 \%$ fecal suspension with the QIAamp ${ }^{\circledR}$ Viral RNA Mini kit (Qiagen, Hilden, Germany) in accordance with the manufacturer's instructions.

For the detection of NoV, reverse transcription PCR (RT-PCR) was performed with the One Step RT-PCR kit (Qiagen, Hilden, Germany) with primers based on the sequence of the norovirus capsid region (Table 1).

To analyze the whole genome sequence of the detected norovirus strain, reverse transcription PCR (RT-PCR) was performed with the One Step RT-PCR kit (Qiagen, Hilden, Germany) with 10 pairs of newly designed primer sets (Table 1). Ten fragments were amplified; eight fragments for ORF1, one fragment for ORF2, and one fragment for ORF3. The PCR products were then analyzed by electrophoresis on $1.5 \%$ agarose gel and ethidium bromide staining. The amplified fragments were purified with HiYield ${ }^{\mathrm{TM}}$ Gel/PCR DNA Extraction kit (RBC, Taipei, Taiwan) from the gel. Then, the products were cloned into the pGEM-T Easy vector (Promega, USA) and were sequenced by Genotech (Daejeon, South Korea).

Sequence data analysis of the composite sequence of ten plasmids aligned with Clustal W method using the
DNAStar software (DNAStar Inc.) revealed that the whole genome was composed of three ORFs, $5100 \mathrm{bp}$ (ORF1), 1623 bp (ORF2), and 807 bp (ORF3).

The dendrograms were constructed with the neighborjoining method. The nucleotide sequence data reported in this study have been deposited into GenBank (accession number; FJ514242).

A comparison of the nucleotide and amino acid sequences of the entire ORF2 with reference strains showed that CUK-3 strain had the highest sequence similarity to the NoV GII-4 reference strain X76717. The nucleotide similarity with CUK-3 was $60.1-88.0 \%$ with the reference strains. A protein database search (BLASTX) for the product of ORF2 yielded $59.3-92.0 \%$ similarity. However, the cut-off value for amino acid similarity between the CUK-3 variant and the GII-4 reference strains showed low identity $(92.0 \%)$ for the genomic sequences (Table 2).

To analyze the genetic relationship between the CUK3 variant and the other variants reported worldwide, the sequences of ORF sequences and the whole genome were processed with multiple sequence alignment. Each analysis of CUK-3 located the ORF1 (5,100 bp) in Cluster I, ORF2 (1623 bp) in Cluster I (2006b), and ORF3 (807 bp) in Cluster I as well as the whole genome sequence (about $5.1 \mathrm{~kb}$ ) in Cluster I (Figure 1).

As shown on the case analysis of ORF1, Cluster I contains Hiroshoma1, Kumamoto1, Saga1, Hokkaodo1, Aomori4, Miayagi5, Akita1, Aichi3 and showed the highest similarity range (98.0 98.9\%) (Figure 1A).

As shown on the case analysis of ORF2, Cluster I (2006b) contains emerged the GII-4 varints in Japan and showed the highest similarity range (97.7 - 99.0\%), whereas Cluster II (2006a) showed low similarity range (91.7 - 92.3\%) (Figure 1B).

As shown on the case analysis of ORF3, Cluster I showed the highest similarity range (97.9 - 99.3\%). Interestingly, 02/03 group contains emerged the GII-4 vaiants in Europe showed more higher similarity range (93.1 - 93.6\%) than Cluster II contains emerged the GII4 variants in Japan (Figure 1C).

As shown on the case analysis of full length, the CUK3 strain showed the highest similarity (98.3\%) with the 2006 epidemic strain, Aichi3/2006/JP strain, whereas Ehime/5-30/2005/JP strain and Lordsdale/1993/UK strain showed the lowest similarity (89.2\%) (Figure 1D).

The CUK-3 strain can be placed on the same branch of the phylogenetic tree as the GII-4/2006b cluster, based on the nucleotide sequence of the RdRp region and the amino acid sequence of the capsid protein (data not shown). Also, the analysis of the GDD motif (AANNTG) of the RdRp region of the GII-4 variants reported worldwide indicated that the GDD motif of CUK-3 strain was similar to that of the GII-4 variants of 
Table 1 Primer sets used in this study

\begin{tabular}{|c|c|c|c|c|}
\hline & Primer & Sequence $\left(5^{\prime} \rightarrow 3^{\prime}\right)$ & Polarity & Region $^{a}$ \\
\hline \multirow[t]{4}{*}{ Diagnosis primer sets } & GIF1M & CTG CCC GAA TTY GTA AAT GAT GAT & + & $5342-5365$ \\
\hline & GIRIM & CCA ACC CAR CCA TTR TAC ATY TG & - & $5649-5671$ \\
\hline & GIIF1M & GGG AGG GCG ATC GCA ATC T & + & $5049-5067$ \\
\hline & GIIRIM & CCR CCI GCA TRI CCR TTR TAC AT & - & 5367-5389 \\
\hline \multirow[t]{20}{*}{ Designed primer sets } & ORF1-1F & GTG AAT GAA GAT GGC GTC TAA C & + & $1-22$ \\
\hline & ORF1-1R & AGT TCC ACT GCA AGG TCC TCA G & - & $999-1020$ \\
\hline & ORF1-2F & CTG AGG ACC TTG CAG TGG AAC T & + & 999-1020 \\
\hline & ORF1-2R & ATG AGG GAA CCA GTG GTG AGA GT & - & 2018-2040 \\
\hline & ORF1-3F & CGT GCT CGA GGC GCA TCG ATT T & + & $1821-1842$ \\
\hline & ORF1-3R & TTG TAC TCA TCA TAC TCT TCA & - & $2701-2721$ \\
\hline & ORF1-4F & CAT TGC TCG AGC ATC AGG GCT AC & + & $2038-2060$ \\
\hline & ORF1-4R & TTG ACT ATC CTC GAC CAG ATG CT & - & $3038-3060$ \\
\hline & ORF1-5F & AGC ATC TGG TCG AGG ATA GTC AA & + & $3038-3060$ \\
\hline & ORF1-5R & GTG GCA CAT ATG ACA GTG TाT CC & - & $3518-3540$ \\
\hline & ORF1-6F & GGA AAC ACT GTC ATA TGT GCC AC & + & $3518-3540$ \\
\hline & ORF1-6R & CAG TCG TTC TTC CGC ATG TGG TGC GG & - & $3935-3960$ \\
\hline & ORF1-7F & CTC AGC ACC AAG ACT AAA TTC TGG A & + & $3635-3659$ \\
\hline & ORF1-7R & TGG GCG ATG GAA TTC CAT TGA GAG G & - & $4485-4509$ \\
\hline & ORF1-8F & ATG GTT AAA TTC TCC CCA GAA CC & + & $4355-4377$ \\
\hline & ORF1-8R & CCA CCT GCA TAA CCA TTG TAC AT & - & $5367-5389$ \\
\hline & ORF2-F & ATG AAG ATG GCG TCG AAT GGC & + & $5085-5105$ \\
\hline & ORF2-R & TTA TAA TGC ACG CCT GCG CCC CGT & - & $6681-6704$ \\
\hline & ORF3-F & ATG GCT GGA GCT TTC TTT GCT & + & $6704-6724$ \\
\hline & ORF3-R & AAA GAC ACT AAA GAA AGG AAA GAT & - & $7532-7555$ \\
\hline
\end{tabular}

${ }^{a}$ Each of sequence number of primer sets region is indicated with Lordsdale strain (X86557) except that of GIF1M and GIR1M set indicated with Norwalk strains (M87661).

Table 2 Nucleotide and amino acid sequence similarities between CUK-3 strain and the full length ORF2 sequences from different Genogroup GII reference strains

\begin{tabular}{|c|c|c|c|c|}
\hline \multirow{2}{*}{$\begin{array}{l}\text { Genotype } \\
\text { Gll-1 }\end{array}$} & \multirow{2}{*}{$\begin{array}{l}\text { Strain } \\
\text { H awaii-USA94 }\end{array}$} & \multirow{2}{*}{$\begin{array}{l}\text { Acession No. } \\
\text { U07611 }\end{array}$} & \multicolumn{2}{|c|}{ Similarity (\%) } \\
\hline & & & $64.9^{a}$ & $64.6^{\mathrm{b}}$ \\
\hline GII-2 & Msham-GBR95 & X81879 & 62.8 & 63.9 \\
\hline Gll-3 & Toronto-CAN93 & U02030 & 64.8 & 67.4 \\
\hline Gll-4 & Bristol-GBR-93 & X76716 & 88.0 & 92.0 \\
\hline Gll-5 & Hilingd-GBRO0 & AJ277607 & 64.6 & 63.9 \\
\hline Gll-6 & Seacrof-GBR00 & AJ277620 & 63.0 & 63.9 \\
\hline GII-7 & Leeds-GBR00 & AJ277608 & 64.3 & 64.8 \\
\hline Gll-8 & Amstdam-NLD99 & AF195848 & 64.3 & 64.8 \\
\hline Gll-9 & VABeach-USA01 & AY038599 & 63.7 & 63.5 \\
\hline Gll-10 & Eufurt-DEU01 & AF421118 & 63.5 & 64.8 \\
\hline Gll-11 & SW918-JPN01 & AB074893 & 62.3 & 64.6 \\
\hline Gll-12 & Wortley-GBR00 & AJ277618 & 63.3 & 63.9 \\
\hline Gll-13 & Faytvil-USA02 & AY113106 & 64.5 & 64.3 \\
\hline Gll-14 & M7-USA03 & AY130761 & 64.3 & 64.8 \\
\hline Gll-15 & J23-USA02 & AY130762 & 60.1 & 59.3 \\
\hline Gll-16 & Tiffin-USA03 & AY502010 & 64.1 & 65.9 \\
\hline Gll-17 & CSE1-USA03 & AY502009 & 63.7 & 65.2 \\
\hline
\end{tabular}

${ }^{a}$ The nucleotide sequences of the ORF2 regions starting at nucleotide 1620 were analyzed.

${ }^{b}$ The amino acid sequences of the ORF2 regions starting at amino acid 540 were analyzed. the epidemic strains in 2006. These strains were common in Hong Kong, Japan, and other regions of North East Asia during the same period $[12,18]$. Such similarities in analyses between CUK-3 and GII-4/2006b strain are evidence of the reemergence of GII-4 variant.

In Europe and Asia, most norovirus outbreaks that occurred after 2002 have been caused by GII-4 variant strains (Figure 2). In addition, biennial emergences of pandemic NoV GII-4 variants have been reported in 2002, 2004, 2006 and 2008 [12,18,19]. These GII-4 variants have also been identified in South Korea and are rapidly spreading worldwide.

Amino acid sequences of the capsid region encoded by ORF2 was compared with a total of 39 GII-4 variants reported from various countries including US, UK, Japan, China, Germany etc. Date of 540 amino acid analyses showed the pattern of changes of two amino acid in first P1 domain, aa238 ( $\mathrm{S}_{\text {GII-4- }>2002} \rightarrow \mathrm{T}_{\text {GII-4- }<2002}$ ), aa250 ( $\mathrm{Y}$

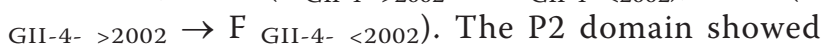
five substitutions of amino acids, aa280 (A GII-4- >2002 $\rightarrow$ $\mathrm{P}_{\text {GII-4- }<2002)}$ ), aa346 (A $\mathrm{A}_{\text {GII-4- }>2002} \rightarrow \mathrm{P}_{\text {GII-4- }<2002)}$ ), aa367 $\left(\mathrm{F}_{\text {GII-4- }}>1995 \rightarrow \mathrm{Y}_{\text {GII-4- }}>2002 \rightarrow \mathrm{F}_{\text {GII-4- }<2002}\right)$, aa376 $\left(\mathrm{Q}_{\text {GII-4- }>2002} \rightarrow \mathrm{E}_{\text {GII-4- }<2002}\right)$, and aa394 (G or S GII-4$\left.>2006 \rightarrow \mathrm{T}_{\mathrm{GII}-4-<2006}\right)$. In addition, the second P1 domain 


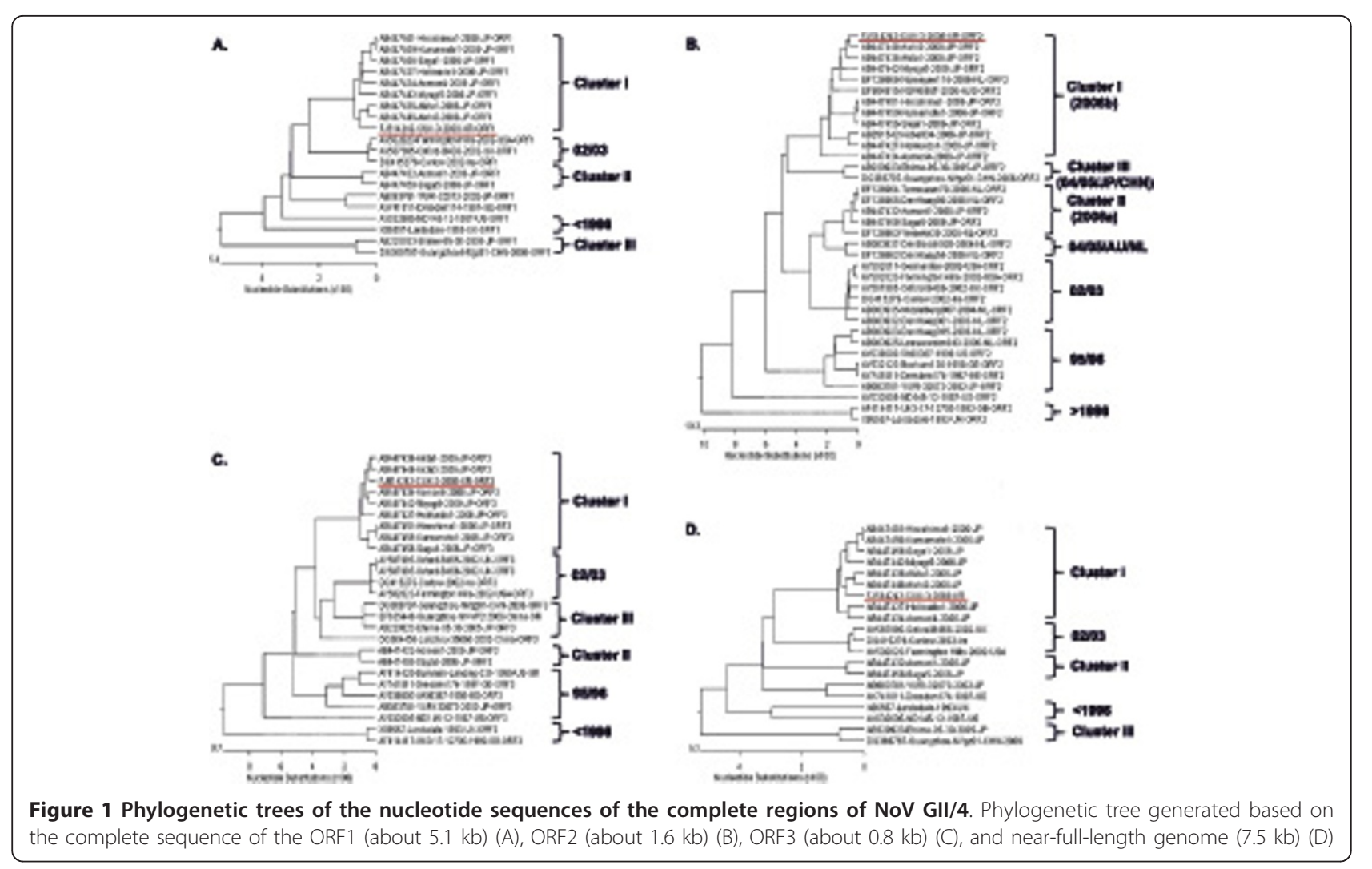

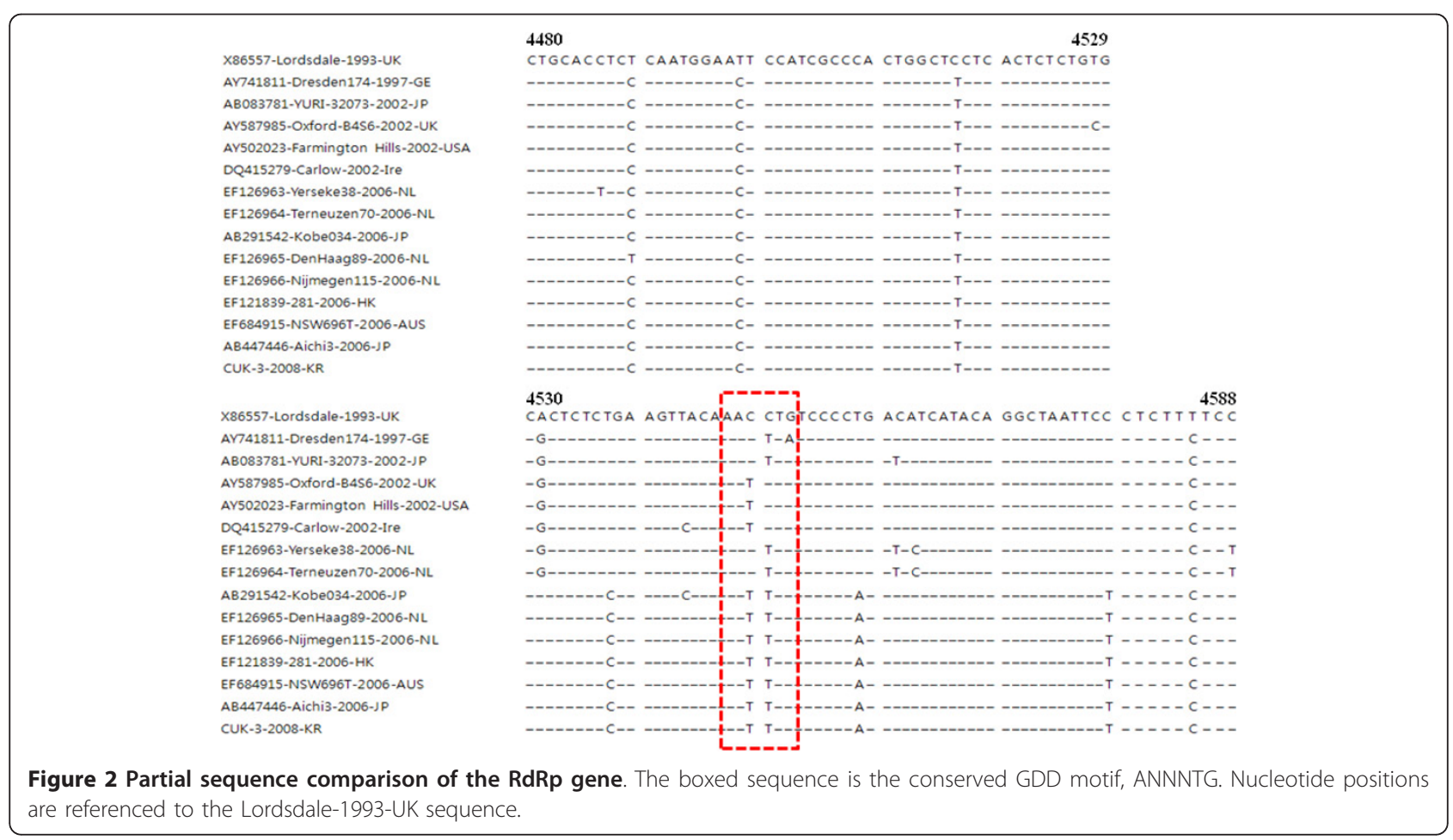


also showed two amino acid substitutions, aa459 (L GII-4$>1995 \rightarrow Q_{\text {GII-4- }>2002}$ ) and aa504 (P GII-4- >2002 $\rightarrow Q_{\text {GII-4- }}$ $<2002$ ). These analytic data showed that there had already been numerous mutations, which lead to the production of a wide range of variants, already before 2002 and produced various variants. And as shown on the case of aa394 of P2 domain which inserted novel amino acid, G or S, and then substituted to $\mathrm{T}$ after 2006 (data not shown). Although previous studies have indicated that the sequence for the NoV capsid region is useful for the diagnosis of NoV variants, no studies have analyzed the RdRp region of the South Korean NoV GII-4 variant. This is the first report that describes the full-length sequence of a NoV GII-4 variant that was isolated from clinical samples in South Korea. We suggest that this sequence can be used as a standard for the comparison of full-length $\mathrm{NoV}$ GII-4 variant sequence with other strains.

The information acquired from whole genome sequencing in this study can be useful not only for a more accurate diagnoses of NoVs but also for the basic research for the elucidation genetic functions. Furthermore, it will be helpful for the prediction of newly appearing pandemic variants through comparison with GII-4 variants in neighboring countries, fundamental research for vaccine development, and eventually, for the field of public health through with the provision of new emerging strains of NoV.

\section{Acknowledgements}

This work was supported by Mid-career Researcher Program (2008-0061575) and Basic Science Research Program (2009-0062720) through NRF grant funded by the MEST.

\section{Author details \\ 'Department of Food and Animal Biotechnology, Department of Agricultural Biotechnology, Center for Agricultural Biomaterials, and Research Institute for Agriculture and Life Sciences, Seoul National University, Seoul 151-921, Republic of Korea. ${ }^{2}$ Department of Microbiology, College of Medicine, The Catholic University of Korea, Seoul 137-701, Republic of Korea. ${ }^{3}$ Department of Microbiology, College of Medicine and Medical Research Institute, Chungbuk National University, Cheongju, Chungcheongbuk-do 361-763, Republic of Korea. ${ }^{4}$ Environmental Infrastructure Research Department, National Institute of Environmental Research, Incheon, 404-708, Republic of Korea.}

\section{Authors' contributions}

SRR and SYP conceived this study. YML, WHJ and designed and conducted the experiments. SGL and JWP analyzed the sequence data and carried out the molecular phylogenetic analysis. SGL and SYP wrote the manuscript. All authors read and approved the final manuscript.

\section{Competing interests}

The authors declare that they have no competing interests.

Received: 28 October 2010 Accepted: 14 April 2011 Published: 14 April 2011

\section{References}

Donaldson, EF, LC Lindesmith, AD Lobue, and RS Baric. 2008. Norovirus pathogenesis: mechanisms of persistence and immune evasion in human populations. Immunol Rev 225: 190-211. doi:10.1111/j.1600-065X.2008.00680. $x$.

Yoon, JS, SG Lee, SK Hong, SA Lee, WH Jheong, SS Oh, MH Oh, GP Ko, CH Lee, and SY Paik. 2008. Molecular epidemiology of norovirus infections in children with acute gastroenteritis in South Korea in November 2005 through November 2006. J Clin Microbiol 46: 1474-1477. doi:10.1128/JCM.02282-07.

Lee, YF, A Nomoto, BM Detjen, and E Wimmer. 1977. A protein covalently linked topoliovirus genome RNA. Proc Natl Acad Sci USA 74: 59-63. doi:10.1073/ pnas.74.1.59.

Rueckert, RR, and E Wimmer. 1984. Systematic nomenclature of picornavirus proteins. J Virol 50: 957-959.

Tan, M, P Huang, J Meller, W Zhong, T Farkas, and X Jiang. 2003. Mutations within the P2 domain of norovirus capsid affect binding to human histoblood group antigens: evidence for a binding pocket. J Virol 77: 12562-12571. doi:10.1128/JVI.77.23.12562-12571.2003.

Nilssson, MK, O Hedlund, M Thorhagen, G Larson, K Johansen, A Ekspong, and L Svensson. 2003. Evolution of human calicivirus RNA in vivo: accumulation of mutations in the protruding P2 domain of the capsid leads to structural changes and possibly a new phenotype. J Virol 77: 13117-13124. doi:10.1128/JVI.77.24.13117-13124.2003.

Lochridge, VP, KI Jutila, JW Graff, and ME Hardy. 2005. Epitopes in the P2 domain of norovirus VP1 recognized by monoclonal antibodies that block cell interactions. J Gen Virol 86: 2799-2806. doi:10.1099/vir.0.81134-0.

Glass, PJ, LJ White, JM Bull, I Leparc-Goffart, ME Hardy, and MK Estes. 2000. Norwalk virus open reading frame 3 encodes aminor structural protein. J Virol 74: 6581-6591. doi:10.1128/JVI.74.14.6581-6591.2000.

Bertolotti-Ciariet, A, SE Crawford, AM Hutson, and MK Estes. 2003. The 3' end of Norwalk virus mRNA contains determinants that regulate the expression and stability of the viral capsid protein VP1: a novel function for the VP2 protein. J Virol 77: 11603-11615. doi:10.1128/JVI.77.21.11603-11615.2003.

Kearney, K, J Menton, and JG Morgan. 2007. Carlow virus, a 2002 Gll-4 variant norovirus strain from Ireland. Virol J 4: 61. doi:10.1186/1743-422X-4-61.

La Rosa, G, M Pourshaban, M laconelli, and M Muscillo. 2008. Detection of genogroup IV noroviruses in environmental and clinical samples and partial sequencing through rapid amplification of CDNA ends. Arch Virol 153: 2077-2083. doi:10.1007/s00705-008-0241-4.

Motomura, K, T Oka, M Yokoyama, H Nakamura, H Mori, H Ode, GS Hansman, K Katayama, T Kanda, T Tanaka, N Takeda, and H Sato. 2008. Identification of monomorphic and divergent haplotypes in the 2006-2007 norovirus GII/4 epidemic population by genome wide tracing of evolutionary history. J Virol 82: 11247-11262. doi:10.1128/JVI.00897-08.

Hale, A, K Mattick, D Lewis, M Estes, X Jiang, J Green, R Eglin, and D Brown. 2000 Distinct epidemiological patterns of Norwalk-like virus infection. J Med Virol 62: 99-103. doi:10.1002/1096-9071(200009)62:13.0.CO;2-0.

Glass, Rl, UD Parashar, and MK Estes. 2009. Norovirus gastroenteritis. N Engl J Med 361: 1776-1785. doi:10.1056/NEJMra0804575.

Lopman, B, H Vennema, E Kohli, P Pothier, A Sanchez, A Negredo, J Buesa, E Schreier, M Reacher, D Brown, J Gray, M Iturriza, C Gallimore, B Bottiger, KO Hedlund, M Torvén, CH von Bonsdorff, L Maunula, M Poljsak-Prijatelj, J Zimsek, G Reuter, G Szücs, B Melegh, L Svennson, Y van Duijnhoven, and M Koopmans. 2004. Increase in viral gastroenteritis outbreaks in Europe and epidemic spread of new norovirus variant. Lancet 363: 682-688. doi:10.1016/ S0140-6736(04)15641-9.

Allen, DJ, R Noad, D Samuel, JJ Gray, P Roy, and M Iturriza-Gómara. 2009. Characterisation of a GII-4 norovirus variant-specific surface-exposed site involved in antibody binding. Virol J 6: 150. doi:10.1186/1743-422X-6-150.

Chung, JY, TH Han, SH Park, SW Kim, and ES Hwang. 2010. Detection of GII-4/ 2006b Variant and Recombinant Noroviruses in Children With Acute Gastroenteritis, South Korea. J Med Virol 82: 146-152. doi:10.1002/jmv.21650.

Ho, EC, PK Cheng, AW Lau, AH Wong, and WW Lim. 2007. A typical norovirus epidemic in Hong Kong during summer of 2006 caused by a new genogroup II/4 variant. J Clin Microbiol 45: 2205-2211. doi:10.1128/ JCM.02489-06.

Tu, ET, RA Bull, GE Greening, J Hewitt, MJ Lyon, JA Marshall, CJ Mclver, WD Rawlinson, and PA White. 2008. Epidemics of gastroenteritis during 2006 were associated with the spread of norovirus Gll.4 variants 2006a and 2006b. Clin Infect Dis 46: 413-420. doi:10.1086/525259.

doi:10.1186/1743-422X-8-167

Cite this article as: Park et al:: Full sequence analysis and

characterization of the South Korean Norovirus GIl-4 variant CUK-3. Virology Journal 2011 8:167. 